\title{
A chromatogram-simplified Streptomyces albus host for heterologous production of natural products
}

\author{
Asif Fazal · Divya Thankachan $\cdot$ Ellie Harris $\cdot$ Ryan F. Seipke $\mathbb{C}$
}

Received: 30 August 2019/Accepted: 14 November 2019/Published online: 28 November 2019

(C) The Author(s) 2019

\begin{abstract}
Cloning natural product biosynthetic gene clusters from cultured or uncultured sources and their subsequent expression by genetically tractable heterologous hosts is an essential strategy for the elucidation and characterisation of novel microbial natural products. The availability of suitable expression hosts is a critical aspect of this workflow. In this work, we mutagenised five endogenous biosynthetic gene clusters from Streptomyces albus S4, which reduced the complexity of chemical extracts generated from the strain and eliminated antifungal and antibacterial bioactivity. We showed that the resulting quintuple mutant can express foreign biosynthetic gene clusters
\end{abstract}

Asif Fazal and Divya Thankachan have contributed equally to this work.

Electronic supplementary material The online version of this article (https://doi.org/10.1007/s10482-019-01360-x) contains supplementary material, which is available to authorized users.

A. Fazal · D. Thankachan · E. Harris · R. F. Seipke $(\square)$ School of Molecular and Cellular Biology, University of Leeds, Leeds LS2 9JT, UK

e-mail: r.seipke@leeds.ac.uk

\section{A. Fazal}

School of Chemistry, University of Leeds, Leeds LS2 9JT, UK

A. Fazal - D. Thankachan - E. Harris - R. F. Seipke Astbury Centre for Structural Molecular Biology, University of Leeds, Leeds LS2 9JT, UK by heterologously producing actinorhodin, cinnamycin and prunustatin. We envisage that our strain will be a useful addition to the growing suite of heterologous expression hosts available for exploring microbial secondary metabolism.

Keywords Streptomyces - Streptomyces albus . Secondary metabolites $\cdot$ Natural products . Heterologous expression

\section{Introduction}

The majority of clinically used antibiotics are derived from natural products produced by Streptomyces species and other closely related Actinobacteria, which were introduced into the clinic during a 'golden era' of antibiotic discovery spanning 1940-1960 (Katz and Baltz 2016). The utility of these agents has been eroded over the last half-century due to misuse. As a consequence, there is now an urgent need to discover new antibiotics to treat bacterial infections, particularly those caused by drug-resistant 'ESKAPE' pathogens (Enterococcus faecium, Staphylococcus aureus, Klebsiella pneumoniae, Acinetobacter baumanii, Pseudomonas aeruginosa, and Enterobacter species) (Boucher et al. 2009). Growing concerns about resistance to antibacterial agents combined with the failure to find new leads from the screening of large 
libraries of synthetic compounds has led to a renewed interest in natural products discovery (Payne et al. 2006). This renaissance has been fuelled to a large extent by the relatively inexpensive cost to sequence genomes of strains that produce promising bioactive small molecules. For example, there are currently $>750$ streptomycete genomes available in GenBank compared to four years ago when there were only $\sim 150$ (Seipke 2015). Analysis of these genome sequences has illuminated the exciting prospect that the majority of microbial secondary metabolism has yet to be uncovered (Doroghazi et al. 2014).

Further study of potentially interesting natural product biosynthetic pathways is often precluded by the poor growth characteristics of the strain and/or genetic intractability. Therefore, a heterologous expression strategy using a faster-growing and genetically tractable host is frequently adopted and indeed has become the method of choice for accessing new natural products and interrogating their biosynthesis. Technology for generating and screening large-insert genomic libraries (e.g. cosmid, BAC and PAC libraries) to obtain biosynthetic gene cluster (BGC) clones are well established (Jones et al. 2013; Qin et al. 2017). These approaches are in the process of being superseded by recombination-based cloning methods for targeted 'capture' of DNA both from isogenic strains (Yamanaka et al. 2014) and environmental DNA (Hover et al. 2018) as well as the ability to assemble natural/synthetic DNA in vivo and in vitro (recently reviewed by (Kim et al. 2015; Luo et al. 2016; Zhang et al. 2016)).

Although fastidiously growing bacterial species, such as Escherichia coli, have been used for some heterologous expression studies, its use has thus far been rather limited because of poor expression of native promoter systems, but in some cases can be overcome by engineered promoter swaps either with the T7 promoter or those recognised by alternative RNA polymerase sigma factors (Stevens et al. 2013; Liu et al. 2015). As a consequence, streptomycetes and other related genera such as Saccharopolyspora and Salinispora are the go-to platform for heterologous expression, because they are metabolically robust with respect to availability of precursors and cofactors, and native promoter elements are more likely to be functional compared to alternative hosts (Zhang et al. 2016; Schniete et al. 2018). Unsurprisingly, well studied species of Streptomyces such as S. avermitilis, $S$. coelicolor, $S$. lividans and $S$. venezuelae are commonly used for heterologous expression studies (Huo et al. 2019; Nepal and Wang 2019). Several of the aforementioned species have been engineered for improved heterologous expression, principally via the mutation of endogenous BGCs (Komatsu et al. 2010, 2013; Gomez-Escribano and Bibb 2011; Thanapipatsiri et al. 2015).

Despite the utility of these strains, $S$. albus is still frequently used as a heterologous expression host. $S$. albus strains are seemingly distributed worldwide and encode a diverse range of natural products produced by a variety of biosynthetic systems (Seipke 2015; Joynt and Seipke 2018). They are genetically tractable and grow relatively quickly using conventional growth media and methodology. S. albus J1074 in particular has served as a workhorse for heterologous production of natural products over the last two decades. Recently, a strain of S. albus J1074 was generated that harbours deletions in 15 of its 22 putative BGCs (Myronovskyi et al. 2018). The resulting strain, which was named Del14, is deficient in the production of several known compounds (i.e. frontalamide, paulomycin, indigoidine, carotenoids, flaviolin, candicidin and antimycin) as well as compounds yet to be characterised (Myronovskyi et al. 2018). Relatively recently, we isolated a streptomycete from leafcutting ants that is closely related to S. albus J1074, which we designated Streptomyces albus S4 (Barke et al. 2010). J1074 and S4 are phylogenetically closely related strains differing in only 12 nucleotide positions across $>12 \mathrm{~kb}$ of DNA sequence representing 29 conserved single-copy conserved phylogenetic markers (Joynt and Seipke 2018). The two strains share $\sim 80 \%$ of their BGCs, but S4 encodes a larger and more diverse complement of secondary metabolites (Seipke 2015). The genetic tractability, robust and fastidious growth and ability to produce diverse secondary metabolites motivated us to modify this strain for easier detection of heterologously produced natural products.

There were two major aims of this work, the first aim was to construct a strain that when cultivated under routine conditions was unable to produce antifungal or antibacterial compounds, and the second aim was to construct a strain from which chemical extracts with reduced complexity could be generated. Here we describe the construction of a chromatogram- 
simplified strain of S. albus $\mathrm{S} 4$ where five BGCs have been mutated and we demonstrate its ability to heterologously produce actinorhodin, cinnamycin and prunustatin.

\section{Materials and methods}

Growth media, strains and reagents

Escherichia coli strains were cultivated using Lennox agar (LA) or broth (LB) and Streptomyces strains were cultured using mannitol-soya flour (MS) agar, tryptic soy broth (TSB) or agar (TSA) (Kieser et al. 2002). Culture media was supplemented with antibiotics as required at the following concentrations: apramycin $(50 \mu \mathrm{g} / \mathrm{ml})$, carbenicillin $(100 \mu \mathrm{g} / \mathrm{ml})$, hygromycin $(75 \mu \mathrm{g} / \mathrm{ml})$, kanamycin $(50 \mu \mathrm{g} / \mathrm{ml})$, nalidixic acid $(25 \mu \mathrm{g} / \mathrm{ml})$. Enzymes were purchased from New England Biolabs and oligonucleotides were purchased from Integrated DNA Technologies. The bacterial strains, cosmids and plasmids used in this study are described in Table S1 and the oligonucleotides are described in Table $\mathrm{S} 2$.

Mutagenesis of biosynthetic gene clusters

In order to delete the ant BGC, the antF gene on S4 Cosmid213 (Seipke et al. 2014) was replaced with the apramycin resistance cassette from pIJ773 using PCR targeted mutagenesis as previously described (Gust et al. 2003). The mutated cosmid was used to generate an apramycin resistant $S$. albus $\mathrm{S} 4 \Delta$ ant $F$ strain. Next, antABCDEFGHIJKLMNO on Cosmid213 were replaced with the apramycin resistance cassette in the same manner as above and subsequently removed by the Flp recombinase, after which the bla gene was replaced with the hygromycin resistance cassette also harbouring an oriT from pIJ10701 as previously described (Gust et al. 2003) to result in Cosmid213 $\triangle$ antFLP B2H. This cosmid was mobilised to $S$. albus S4 $\Delta a n t F$ and a single hygromycin resistant transconjugant was selected and subsequently passaged twice in the absence of selection prior to replica plate identification of an apramycin sensitive and hygromycin sensitive isolate that we named S. albus S4 $\Delta 1$. The integrity of this strain was confirmed by PCR using primers RFS236 and RFS237.
The can BGC was mutated using the previously constructed fscC (STRS4_02234) deletion plasmid, pKC1132-UpDn (Seipke et al. 2011a). pKC1132UpDn was mobilised to $S$. albus $\mathrm{S} 4 \Delta 1$ strain and a single apramycin resistant transconjugant was selected and passaged as above until an apramycin-sensitive isolate was identified, which we named $S$. albus $\mathrm{S} 4 \Delta 2$.

The surugamide, fredericamycin and albaflavenone BGCs were mutated using the pCRISPomyces-2 system describe previously (Cobb et al. 2015). First, a single-guide RNA protospacer was generated by annealing oligonucleotides EH_S9 and EH_S10 (surugamide), EH_S3 and EH_S4 (albaflavenone) and EH_S7 and EH_S8 (fredericamycin); the resulting DNA fragments were cloned into the BbsI site of pCRISPomyces- 2 by Golden Gate Assembly. Second, two overlapping DNA fragments representing a homology-directed repair template were generated by PCR using the primers listed in Table S2. The overlapping PCR products were subsequently cloned into the $\mathrm{XbaI}$ site of protospacer-containing pCRISPomyces-2 plasmid using the NEBbuilder HiFi DNA assembly kit. The resulting CRISPR/Cas9 editing plasmids, pCRISPomyces-2-sur, pCRISPomyces-2alb, pCRISPomyces-2-fdm were sequentially mobilised to Streptomyces and cured as described previously (McLean et al. 2016) and schematically illustrated in Figure $\mathrm{S} 1$ to result in the quintuple mutant strain described in this study, S. albus S4 $\Delta 5$.

Genome sequencing and bioinformatics analysis

S. albus $\mathrm{S} 4 \Delta 5$ chromosomal DNA was sequenced by Microbes NG (Birmingham, UK) using the Illumina MiSeq platform. This resulted in the generation of $2,859,380$ paired-end reads that were $250 \mathrm{nt}$ in length. $2,809,652$ of these reads were mapped the S. albus S4 wild-type chromosome [GenBank accession CADY00000000.1 (Seipke et al. 2011b)] using the Geneious assembler (version R8.1.19). Schematic representations of relevant loci depicted in Figs. S2S6 were generated using Geneious version R8.1.19.

Bioactivity assays

Indicator organism was cultivated overnight in LB at $37{ }^{\circ} \mathrm{C}$. Overnight culture was diluted to an $\mathrm{OD}_{625 \mathrm{~nm}}$ of 0.08 in LB and spread onto an LB agar plate using a rotary platform. Sterile paper discs (6 $\mathrm{mm}$ diameter) 


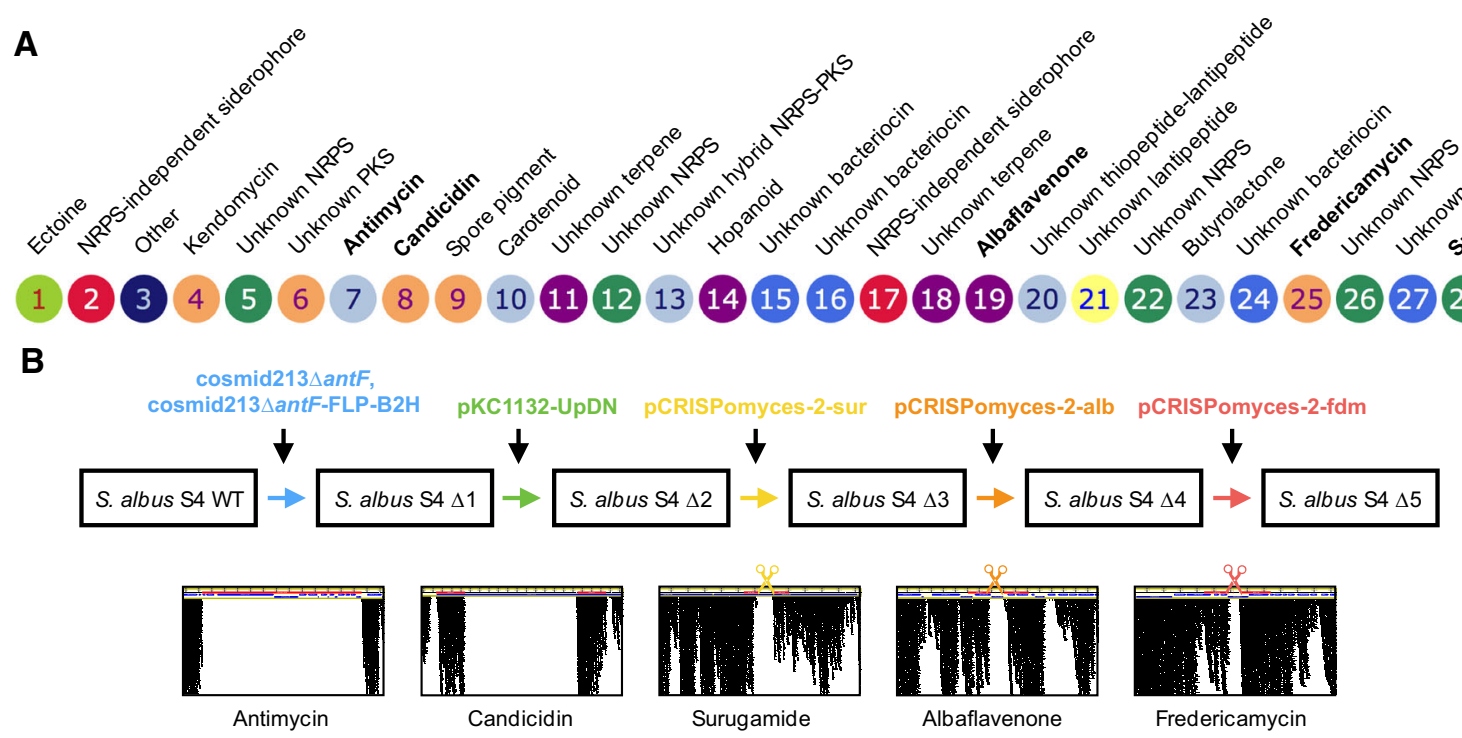

Fig. 1 Streptomyces albus S4 biosynthetic gene clusters (BGCs) and their mutation. a Diagrammatic representation of putative BGCs identified by antiSMASH 3.0. The BGCs are numbered and the experimentally determined or bioinformatically deduced products are listed where known. BGCs targeted for mutagenesis in this study are indicated by bold text. b Schematic of the workflow used to create S. albus S4 $\Delta 5$.

holding $60 \mu \mathrm{l}$ of chemical extract were placed atop seeded indicator plates, which were subsequently incubated at $37^{\circ} \mathrm{C}$ (Micrococcus luteus) or room temperature (Candida albicans) and visualized the following day.

HPLC analysis

S. albus strains were cultivated in liquid MS $(50 \mathrm{ml})$ whilst shaking at $220 \mathrm{rpm}$ in a $250 \mathrm{ml}$ flask at $30{ }^{\circ} \mathrm{C}$ for 7 days. Bacterial cells and solid matter from the culture medium were removed by centrifugation and $45 \mathrm{ml}$ of supernatant were extracted with $90 \mathrm{ml}$ of ethyl acetate. The extract was evaporated to dryness under reduced pressure and resuspended in $100 \%$ methanol $(500 \mu \mathrm{l})$. The methanolic extract was centrifuged for $10 \mathrm{~min}$ at $16,000 \times g$ to remove insoluble material prior to sample injection $(10 \mu \mathrm{l})$ into a Dionex HPLC instrument. Compounds were separated on a Phenomenex Luna C18 column (5 $\mu \mathrm{m}$, $150 \times 4.6 \mathrm{~mm}$ ) using the following gradient (solvent A: $5 \%$ acetonitrile, $0.1 \%$ formic acid, solvent B: $95 \%$ acetonitrile, $0.05 \%$ formic acid, flow rate $1 \mathrm{ml} / \mathrm{min}$ ):
Knockout constructs are colour coded according to their targeted mutation and plasmid and strain designations are described in Table S1. The bottom panel depicts Illumina MiSeq reads mapped to the relevant locus of the $S$. albus $\mathrm{S} 4 \Delta 5$ genome illustrating that the desired mutation was achieved. The scissors indicate cleavage by the Cas 9 nuclease. Full size versions of these images are shown in Figures S1-S5. (Color figure online)

0-40 min, $\quad 0-100 \% \quad \mathrm{~B} ; \quad 40-43 \mathrm{~min}, \quad 100 \% \quad \mathrm{~B}$; 43-51 $\min 0 \% \mathrm{~B}$.

\section{LCMS analysis}

Clones of the actinorhodin, cinnamycin and neoantimycin BGCs were introduced into $S$. albus $\Delta 5$ by intergeneric conjugation from Escherichia coli ET12567/pUZ8002. Apramycin resistant transconjugants were verified to have received BGCs by PCR. For the production of actinorhodin and prunustatin, MS agar plates (pH 6.7) were seeded with either $\Delta 5$ or $\Delta 5 /$ Act or $\Delta 5 /$ Prun and cultivated for 7 days at $30{ }^{\circ} \mathrm{C}$. The agar was then cut into small rectangular pieces and placed into an Erlenmeyer flask and metabolites extracted with ethyl acetate $(100 \mathrm{ml})$ for $24 \mathrm{~h}$. The ethyl acetate extract was decanted and concentrated in vacuo and the dried residue was resuspended in $100 \%$ methanol $(500 \mu \mathrm{l})$. For the production of cinnamycin, S. albus S4 $\Delta 5$ and $\Delta 5 /$ Cin strains were cultured in $50 \mathrm{ml} \mathrm{TSB}\left(\mathrm{pH} 7.0\right.$ ) for 7 days at $30^{\circ} \mathrm{C}$. The resulting mycelia was collected by centrifugation and extracted with $100 \%$ methanol $(45 \mathrm{ml})$ for $4 \mathrm{~h}$. The methanolic extracts were evaporated to dryness in vacuo and 
resuspended in $100 \%$ methanol $(500 \mu \mathrm{l})$ as above. Equal volumes of methanolic extract for three independently cultivated replicates of $\Delta 5, \Delta 5 /$ Act, $\Delta 5 / \mathrm{Cin}$, $\Delta 5 /$ Prun were pooled and centrifuged for $10 \mathrm{~min}$ at $16,000 \times g$ to remove insoluble material prior to analysis by high resolution electrospray ionization liquid chromatography mass spectrometry (LCHRMS). Only the supernatant $(2 \mu \mathrm{l})$ was injected into a Bruker MaXis Impact TOF mass spectrometer and equipped with a Dionex Ultimate 3000 HPLC exactly as previously described (Liu et al. 2015).

\section{A}

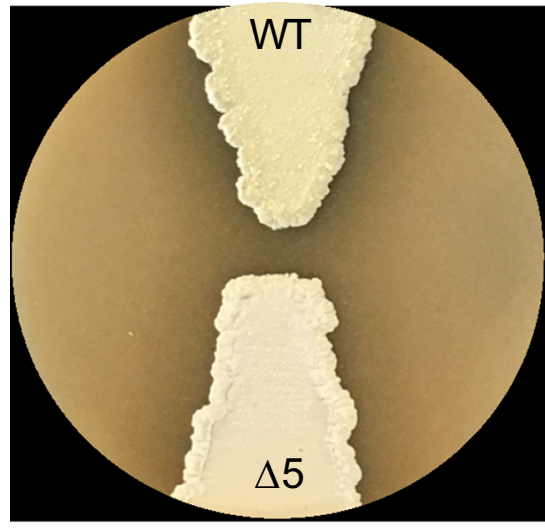

C

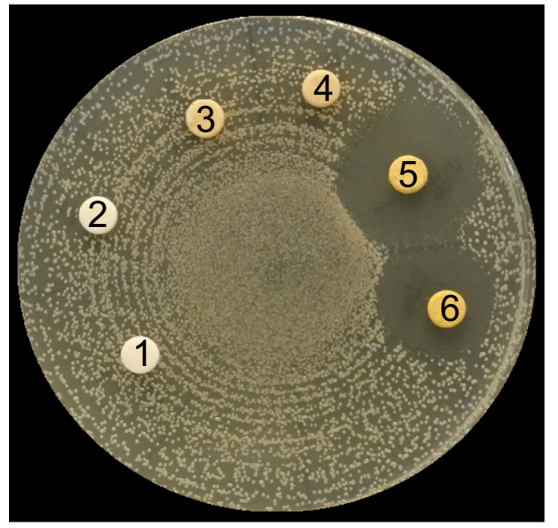

Fig. 2 S. albus S4 $\Delta 5$ sporulates normally and produces biomass equal to that of the WT strain during growth in liquid media. a Sporulation of S. albus strains after growth on MS agar for 7 days. b Wet cell mass of $S$. albus strains originating from TSB cultures. The values reported are the means for a $20 \mathrm{ml}$ sample of culture and the vertical bars represent the standard error of the mean $(n=3)$. The results are not statistically significantly different in a Student's $t$ test with a $P$ value $>0.60$.

\section{Results and Discussion}

\section{Construction of Streptomyces albus S4 $\Delta 5$}

The $S$. albus $\mathrm{S} 4$ genome was previously sequenced (Seipke et al. 2011a, b) and it harbours at least 28 putative natural product biosynthetic gene clusters (BGCs) according to antiSMASH 3.0 (Fig. 1a) (Weber et al. 2015). Chemical extracts prepared from $S$. albus $\mathrm{S} 4$ cultivated under a variety of culture conditions are dominated by antimycins and candicidin (Barke et al. 2010; Seipke et al. 2011a). Therefore, abolishing production of these compounds was a top priority in our mutagenesis strategy. With the longerterm goal of ultimately developing an S. albus host as a platform to screen uncharacterised BGC clones for
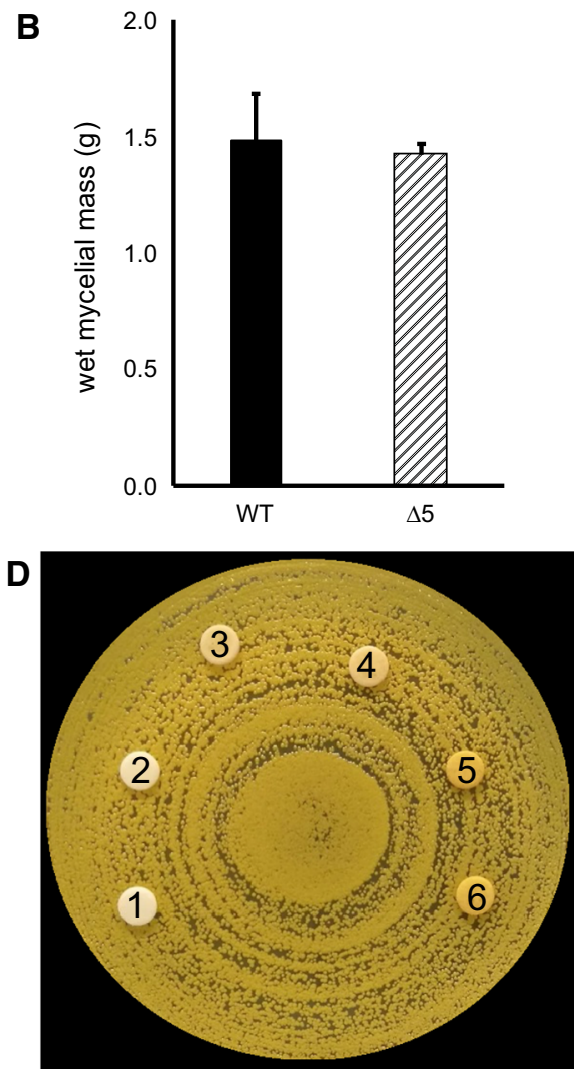

Bioactivity of ethyl acetate chemical extracts generated from $S$. albus strains cultivated in liquid MS against $\mathbf{c}$ Candida albicans and $\mathbf{d}$ Micrococcus luteus. The discs on each plate are numbered and are as follows: 1, methanol; 2, extract from MS media; 3 and $4, S$. albus $\Delta 5$ extract; 5 and 6, S. albus S4 WT extract. A zone of inhibited growth is apparent only for the wild-type extract against $C$. albicans 
antibacterial activity, we also targeted the albaflavenone, surugamide and fredericamycin BGCs because these compounds have been reported to have antibacterial properties (Warnick-Pickle et al. 1981; Gürtler et al. 1994; Wang et al. 2014). We mutated these five BGCs using conventional marker exchange mutagenesis (candicidin), PCR-targeted recombineering (antimycin) and CRISPR/Cas9 genome editing (albaflavenone, surugamide and fredericamycin). The workflow for this strategy is presented in Fig. 1b and is described in the materials and methods section. The final mutant strain, which we designated S. albus $\mathrm{S} 4 \Delta 5$, harbours a complete pathway deletion in the antimycin BGC, and deletions in key biosynthetic genes for the other four targeted BGCs. Deletions were first verified by PCR and the overall integrity of the $S$. albus $\mathrm{S} 4 \Delta 5$ strain was verified by genome sequencing using the Illumina MiSeq platform. The expected mutations were verified by mapping sequence reads to the S. albus S4 wild-type genome sequence (Seipke et al. 2011b). A schematic representation of the relevant areas of the mapping are displayed in Fig. 1b and Figs. S1-S5.

Sporulation, biomass and bioactivity of $\Delta 5$

In order to ensure that serial passage (e.g. to cure plasmids with temperature-sensitive replicons such as that employed by pCRISPomyces-2) did not lead to mutation(s) adversely impacting sporulation and/or growth of $\Delta 5$, we assessed sporulation on MS agar and analysed its ability to produce biomass when cultivated using TSB. The $\Delta 5$ mutant strain sporulated equally as well as the wild-type strain, which indicated the absence of mutations deleterious to a normal developmental cycle and it also produced a comparable abundance of dispersed biomass to that of its parent (Fig. 2a, b). Next, we analysed the bioactivity of ethyl acetate chemical extracts prepared from culture supernatants of $S$. albus $\mathrm{S} 4$ wild-type or $\Delta 5$ cultivated in liquid MS against Candida albicans and Micrococcus luteus, which revealed that extracts from the $\Delta 5$ strain were not bioactive (Fig. 2c, d).

Chromatographic profile of $\Delta 5$

Chemical extracts generated from S. albus S4 wildtype are complex and chromatographs are dominated by candicidins and antimycins when using a standard aqueous/organic phase gradient of 5-95\% acetonitrile or methanol (Seipke et al. 2011a, 2014). In order to assess to what extent this complexity was reduced in extracts generated from the $\Delta 5$ mutant, we cultivated S4 wild-type and $\Delta 5$ using liquid MS and performed an ethyl acetate extraction of the resulting clarified culture supernatant. Next, extracts were analysed by HPLC, which as anticipated, revealed that the extracts generated from the $\Delta 5$ were far less complex than those generated from the wild-type strain (Fig. 3). The cleaner chromatographic background of the $S$. albus S4 $\Delta 5$ strain will simplify detection of heterologously produced natural products by HPLC or LC-MS and ease their subsequent purification.

Heterologous production of actinorhodin, cinnamycin and prunustatin

As a proof of principle to demonstrate that $S 4 \Delta 5$ can produce natural products encoded by other taxa, we introduced three foreign BGCs: the actinorhodin BGC from $S$. coelicolor A3(2), the cinnamycin BGC from $S$. cinnamoneus DSM 40,646 and the neoantimycin/

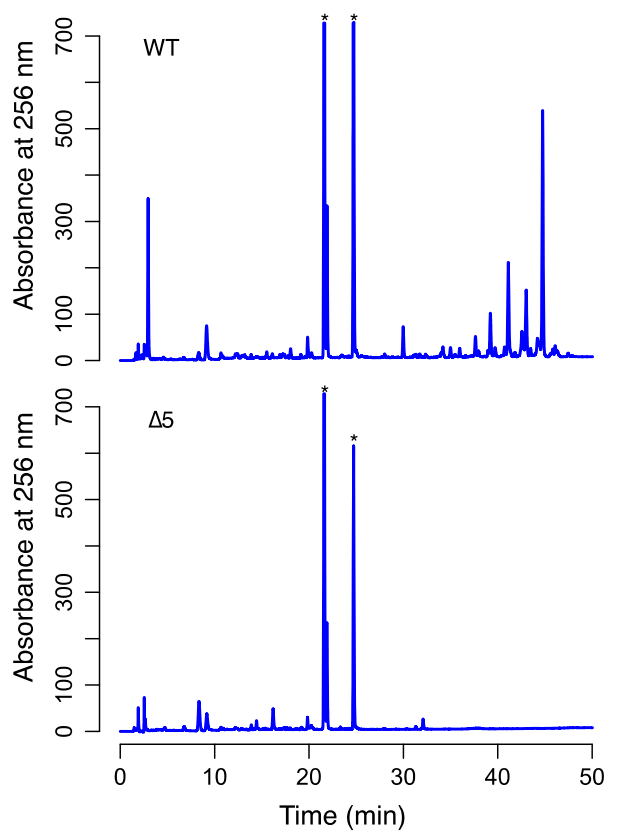

Fig. 3 HPLC analysis of ethyl acetate extracts prepared from $S$. albus S4 WT and $\Delta 5$ strains. The HPLC chromatogram originating from the $\Delta 5$ strain is simpler in composition compared to that of the WT strain. The asterisks indicate HPLC peaks that are not fully visible in this image, but can be seen in Fig. S6 
Fig. 4 Heterologous production of $\gamma$ actinorhodin (1), cinnamycin (2) and prunustatin (3) by $S$. albus S4 $\Delta 5$. LC-HRMS analysis of chemical extracts prepared from the indicated strains. The $m / z$ values corresponding to the $[\mathrm{M}+2 \mathrm{H}]^{2+}$ ions derived from $\gamma$-actinorhodin $\left(\mathrm{C}_{32} \mathrm{H}_{22} \mathrm{O}_{14}\right)$, cinnamycin $\left(\mathrm{C}_{89} \mathrm{H}_{125} \mathrm{~N}_{25} \mathrm{O}_{25} \mathrm{~S}_{3}\right)$, and prunustatin $\left(\mathrm{C}_{36} \mathrm{H}_{44} \mathrm{~N}_{2} \mathrm{O}_{12}\right)$ are shown. The intensity scale for extracted ion chromatograms is $1 \times 104$ for $\gamma$-actinorhodin and cinnamycin, and $1 \times 105$ for prunustatin
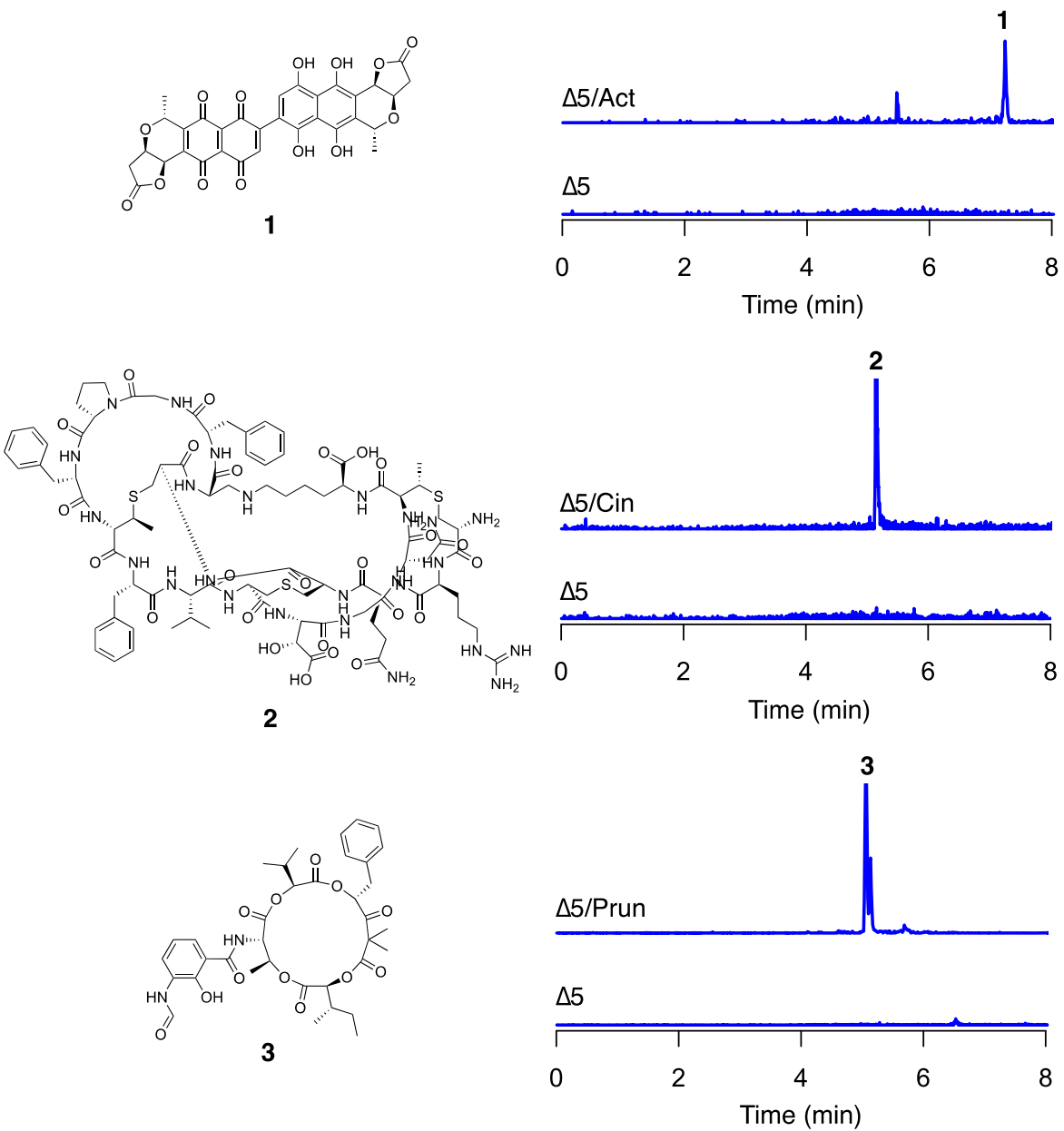

prunustatin BGC from $S$. orinoci B-NRRL 3379 (Widdick et al. 2003; Gomez-Escribano and Bibb 2011; Skyrud et al. 2018). These BGCs were selected in part due to accessibility, but also because they comprised three different biosynthetic systems. Actinorhodin is a type II polyketide antibacterial, cinnamycin is a ribosomally synthesised and posttranslationally modified peptide antibacterial and neoantimycin/prunustatin is a hybrid non-ribosomal peptide / polyketide anti-cancer compound. Recombinant strains harbouring these BGCs were generated and cultivated using MS agar or TSB for seven days. Next, chemical extracts were prepared from the $\Delta 5 /$ Act, $\Delta 5 /$ Cin, $\Delta 5 /$ Prun strains and their parent and subsequently analysed by LC-HRMS (Fig. 4). Inspection of the resulting data revealed the presence of compounds consistent with $\gamma$-actinorhodin, cinnamycin and prunustatin in chemical extracts prepared from $\Delta 5 /$ Act, $\Delta 5 /$ Cin, $\Delta 5 /$ Prun, respectively and importantly, their absence from the $\Delta 5$ parental strain. These data indicate that $S$. albus $\mathrm{S} 4 \Delta 5$ is capable of heterologous expressing foreign BGCs from classes of varying biosynthetic logic.

\section{Summary and concluding perspectives}

We have constructed a strain of S. albus S4 in which the antimycin, candicidin, albaflavenone, surugamide and fredericamycin BGCs have been mutated. We showed that the resulting strain, S. albus S4 $\Delta 5$, possessed a simpler chromatographic profile and did not possess antibacterial or antifungal activity using the growth media tested and also demonstrated that it could serve as a surrogate host for the production of actinorhodin, cinnamycin and prunustatin. 
Interestingly, during the course of our study we also tried to heterologously produce bicyclomycin and methylenomycin, but were unable to detect either metabolite by LC-HRMS. The unsuccessful attempt to heterologously produce bicyclomycin and methylenomycin underscores a key consideration for heterologous expression studies - host selection. For instance, $S$. coelicolor is a native producer of methylenomycin and was recently used as a host for bicyclomycin production (Vior et al. 2018), however it will not produce neoantimycin/prunustatin unless the biosynthetic genes are artificially expressed from constitutive promoters (Skyrud et al. 2018). Indeed, there is no panacea in terms of a heterologous expression host, each one being somewhat enigmatic with peculiarities rooted in primary metabolism (i.e. precursor and cofactor supply) or promoter recognition elements. Fortuitously, there are now greater flexibility than ever before in terms of host selection. For instance, a suite of $S$. coelicolor M145 $\Delta a c t \Delta r e d \Delta c d a \Delta c p k$ variants (M1146, M1152, M1154) are a commonly used (Gomez-Escribano and Bibb 2011). Additionally, the endogenous BGC content of $S$. avermitilis was also reduced to enhance production and detection of heterologous compounds (Komatsu et al. 2010, 2013) and an impressive 15 BGCs were recently removed from S. albus $\mathrm{J} 1074$.

(Myronovskyi et al. 2018). It is therefore possible, and indeed advantageous and wise when pursuing heterologous expression of a BGC to cast a wide net and screen multiple hosts. The S. albus S4 $\Delta 5$ strain constructed here will hopefully be a useful tool to that end.

Acknowledgements We are grateful to Iain Manfield for assistance with HPLC experiments, which were conducted in the University of Leeds Biomolecular Interactions Facility. We thank David Widdick and Mervyn Bibb for providing the cinnamycin and actinorhodin BGC clones, Andy Truman for providing the bicyclomycin BGC clone and Christophe Corre for providing the methylenomycin BGC clone. This work was supported by Biotechnology and Biological Sciences Research Council responsive mode Grant BB/N007980/1 awarded to RFS. AF and DT were funded by $\mathrm{PhD}$ studentships funded by the University of Leeds.

\section{Compliance with ethical standards}

Conflict of interest The authors declare that there are no conflicts of interest and that the funders had no role in study design, data collection and interpretation, or the decision to submit the work for publication.
Open Access This article is distributed under the terms of the Creative Commons Attribution 4.0 International License (http:// creativecommons.org/licenses/by/4.0/), which permits unrestricted use, distribution, and reproduction in any medium, provided you give appropriate credit to the original author(s) and the source, provide a link to the Creative Commons license, and indicate if changes were made.

\section{References}

Barke J, Seipke RF, Grüschow S, Heavens D, Drou N, Bibb MJ, Goss RJM, Yu DW, Hutchings MI (2010) A mixed community of actinomycetes produce multiple antibiotics for the fungus farming ant Acromyrmex octospinosus. BMC Biol 8:109. https://doi.org/10.1186/1741-7007-8-109

Boucher HW, Talbot GH, Bradley JS, Edwards JE, Gilbert D, Rice LB, Scheld M, Spellberg B, Bartlett J (2009) Bad bugs, no drugs: no ESKAPE! An update from the Infectious Diseases Society of America. Clin Infecti Dis 48:1-12. https://doi.org/10.1086/595011

Cobb RE, Wang Y, Zhao H (2015) High-efficiency multiplex genome editing of Streptomyces species using an engineered CRISPR/Cas system. ACS Synth Biol 4:723-728. https://doi.org/10.1021/sb500351f

Doroghazi JR, Albright JC, Goering AW, Haines RR, Tchalukov KA, Labeda DP, Metcalf WW (2014) A roadmap for natural product discovery based on large-scale genomics and metabolomics. Nat Chem Biol 10:963-968. https://doi. org/10.1038/nchembio. 1659

Gomez-Escribano JP, Bibb MJ (2011) Engineering Streptomyces coelicolor for heterologous expression of secondary metabolite gene clusters. Microbial Biotechnol 4:207-215. https://doi.org/10.1111/j.1751-7915.2010.00219.x

Gürtler H, Pedersen R, Anthoni U, Christophersen C, Nielsen PH, Wellington EM, Pedersen C, Bock K (1994) Albaflavenone, a sesquiterpene ketone with a zizaene skeleton produced by a streptomycete with a new rope morphology. J Antibiot 47:434-439

Gust B, Challis GL, Fowler K, Kieser T, Chater KF (2003) PCRtargeted Streptomyces gene replacement identifies a protein domain needed for biosynthesis of the sesquiterpene soil odor geosmin. Proc Natl Acad Sci USA 100:1541-1546. https://doi.org/10.1073/pnas.0337542100

Hover BM, Kim S-H, Katz M, Charlop-Powers Z, Owen JG, Ternei MA, Maniko J, Estrela AB, Molina H, Park S, Perlin DS, Brady SF (2018) Culture-independent discovery of the malacidins as calcium-dependent antibiotics with activity against multidrug-resistant Gram-positive pathogens. Nat Microbiol 3:1-10. https://doi.org/10.1038/s41564-0180110-1

Huo L, Hug JJ, Fu C, Bian X, Zhang Y, Müller R (2019) Heterologous expression of bacterial natural product biosynthetic pathways. Nat Prod Rep 4:206-225. https:// doi.org/10.1039/C8NP00091C

Jones AC, Gust B, Kulik A, Heide L, Buttner MJ, Bibb MJ (2013) Phage P1-Derived Artificial chromosomes facilitate heterologous expression of the FK506 gene cluster. PLoS 
ONE 8:e69319-e69329. https://doi.org/10.1371/journal. pone.0069319

Joynt R, Seipke RF (2018) A phylogenetic and evolutionary analysis of antimycin biosynthesis. Microbiology 164:28-39. https://doi.org/10.1099/mic.0.000572

Katz L, Baltz RH (2016) Natural product discovery: past, present, and future. J Indian Microbiol Biotechnol 43:155-176. https://doi.org/10.1007/s10295-015-1723-5

Kieser TB, Buttner MJ, Chater KF, Hopwood DA (2002) Practical Streptomyces genetics. The John Innes Foundation, Norwich

Kim E, Moore BS, Yoon YJ (2015) Reinvigorating natural product combinatorial biosynthesis with synthetic biology. Nat Chem Biol 11:649-659. https://doi.org/10.1038/ nchembio. 1893

Komatsu M, Uchiyama T, Omura S, Cane DE, Ikeda H (2010) Genome-minimized Streptomyces host for the heterologous expression of secondary metabolism. Proc Natl Acad Sci USA 107:2646-2651. https://doi.org/10.1073/pnas. 0914833107

Komatsu M, Komatsu K, Koiwai H, Yamada Y, Kozone I, Izumikawa M, Hashimoto J, Takagi M, Omura S, Shin-ya K, Cane DE, Ikeda H (2013) Engineered Streptomyces avermitilis host for heterologous expression of biosynthetic gene cluster for secondary metabolites. ACS Synth Biol 2:384-396. https://doi.org/10.1021/sb3001003

Liu J, Zhu X, Seipke RF, Zhang W (2015) Biosynthesis of antimycins with a reconstituted 3-formamidosalicylate pharmacophore in Escherichia coli. ACS Synth Biol 4:559-565. https://doi.org/10.1021/sb5003136

Luo Y, Enghiad B, Zhao H (2016) New tools for reconstruction and heterologous expression of natural product biosynthetic gene clusters. Nat Prod Rep 33:174-182. https://doi. org/10.1039/C5NP00085H

McLean TC, Hoskisson PA, Seipke RF (2016) Coordinate regulation of antimycin and candicidin biosynthesis. mSphere 1:e00305-16. https://doi.org/10.1128/mSphere. 00305-16

Myronovskyi M, Rosenkränzer B, Nadmid S, Pujic P, Normand P, Luzhetskyy A (2018) Generation of a cluster-free Streptomyces albus chassis strains for improved heterologous expression of secondary metabolite clusters. Metab Eng 49:316-324. https://doi.org/10.1016/j.ymben.2018. 09.004

Nepal KK, Wang G (2019) Streptomycetes_Surrogate hosts for the genetic manipulation of biosynthetic gene clusters and production of natural products. Biotechnol Adv 37:1-20. https://doi.org/10.1016/j.biotechadv.2018.10.003

Payne DJ, Gwynn MN, Holmes DJ, Pompliano DL (2006) Drugs for bad bugs: confronting the challenges of antibacterial discovery. Nat Rev Drug Discov 6:29-40. https://doi.org/10.1038/nrd2201

Qin Z, Munnoch JT, Devine R, Holmes NA, Seipke RF, Wilkinson KA, Wilkinson B, Hutchings MI (2017) Formicamycins, antibacterial polyketides produced by Streptomyces formicae isolated from African Tetraponera plant-ants. Chem Sci 8:3218-3227. https://doi.org/10. 1039/c6sc04265a

Schniete JK, Cruz-Morales P, Selem Mojica N, FernándezMartínez LT, Hunter IS, Barona-Gómez F, Hoskisson PA (2018) Expanding primary metabolism helps generate the metabolic robustness to facilitate antibiotic biosynthesis in Streptomyces. mBio 9:e02283-17-13. https://doi.org/10. 1128/mBio.02283-17

Seipke RF (2015) Strain-level diversity of secondary metabolism in Streptomyces albus. PLoS ONE 10:e0116457e116514. https://doi.org/10.1371/journal.pone.0116457

Seipke RF, Barke J, Brearley C, Hill L, Yu DW, Goss RJM, Hutchings MI (2011a) A Single Streptomyces symbiont makes multiple antifungals to support the fungus farming ant Acromyrmex octospinosus. PLoS ONE 6:e22028e22038. https://doi.org/10.1371/journal.pone.0022028

Seipke RF, Crossman L, Drou N, Heavens D, Bibb MJ, Caccamo M, Hutchings MI (2011b) Draft genome sequence of Streptomyces strain S4, a symbiont of the leaf-cutting ant Acromyrmex octospinosus. J Bacteriol 193:4270-4271. https://doi.org/10.1128/JB.05275-11

Seipke RF, Patrick E, Hutchings MI (2014) Regulation of antimycin biosynthesis by the orphan ECF RNA polymerase sigma factor $\sigma^{\text {AntA }}$. PeerJ 2:e253. https://doi.org/ 10.7717/peerj.253

Skyrud W, Liu J, Thankachan D, Cabrera M, Seipke RF, Zhang W (2018) Biosynthesis of the 15-membered ring depsipeptide neoantimycin. ACS Chem Biol 13:1398-1406. https://doi.org/10.1021/acschembio.8b00298

Stevens DC, Conway KR, Pearce N, Villegas-Peñaranda LR, Garza AG, Boddy CN (2013) Alternative sigma factor over-expression enables heterologous expression of a type II polyketide biosynthetic pathway in Escherichia coli. PLoS ONE 8:e64858. https://doi.org/10.1371/journal. pone. 0064858

Thanapipatsiri A, Claesen J, Gomez-Escribano JP, Bibb M, Thamchaipenet A (2015) A Streptomyces coelicolor host for the heterologous expression of Type III polyketide synthase genes. Microb Cell Fact 14:1-11. https://doi.org/ 10.1186/s12934-015-0335-0

Vior NM, Lacret R, Chandra G, Dorai-Raj S, Trick M, Truman AW (2018) Discovery and biosynthesis of the antibiotic bicyclomycin in distantly related bacterial classes. Appl Environ Microbiol 84:e02828-e2917. https://doi.org/10. 1128/AEM.02828-17

Wang X, Shaaban KA, Elshahawi SI, Ponomareva LV, Sunkara M, Copley GC, Hower JC, Morris AJ, Kharel MK, Thorson JS (2014) Mullinamides A and B, new cyclopeptides produced by the Ruth Mullins coal mine fire isolate Streptomyces sp. RM-27-46. J Antibiot 67:571-575. https://doi. org/10.1038/ja.2014.37

Warnick-Pickle DJ, Byrne KM, Pandey RC, White RJ (1981) Fredericamycin A, a new antitumor antibiotic. II. Biological properties. J Antibiot 34:1402-1407

Weber T, Blin K, Duddela S, Krug D, Kim HU, Bruccoleri R, Lee SY, Fischbach MA, Müller R, Wohlleben W, Breitling R, Takano E, Medema MH (2015) antiSMASH 3.0-a comprehensive resource for the genome mining of biosynthetic gene clusters. Nucleic Acids Res 43:W237W243. https://doi.org/10.1093/nar/gkv437

Widdick DA, Dodd HM, Barraille P, White J, Stein TH, Chater KF, Gasson MJ, Bibb MJ (2003) Cloning and engineering of the cinnamycin biosynthetic gene cluster from Streptomyces cinnamoneus cinnamoneus DSM 40005. Proc Natl Acad Sci USA 100:4316-4321. https://doi.org/10.1073/ pnas.0230516100 
Yamanaka K, Reynolds KA, Kersten RD, Ryan KS, Gonzalez DJ, Nizet V, Dorrestein PC, Moore BS (2014) Direct cloning and refactoring of a silent lipopeptide biosynthetic gene cluster yields the antibiotic taromycin A. Proc Natl Acad Sci USA 111:1957-1962. https://doi.org/10.1073/ pnas.1319584111

Zhang MM, Wang Y, Ang EL, Zhao H (2016) Engineering microbial hosts for production of bacterial natural products. Nat Prod Rep 00:1-25. https://doi.org/10.1039/ C6NP00017G

Publisher's Note Springer Nature remains neutral with regard to jurisdictional claims in published maps and institutional affiliations. 